\title{
Fine lattice simulations with chirally symmetric fermions
}

\section{J. Noaki ${ }^{*, a}$, S. Aoki ${ }^{b, c}$, G. Cossu ${ }^{a}$, H. Fukaya ${ }^{d}$, S. Hashimoto ${ }^{a, e}$, T. Kaneko ${ }^{a, e}$ for JLQCD Collaboration}

${ }^{a}$ High Energy Accelerator Research Organization (KEK), Tsukuba 305-0801, Japan

${ }^{b}$ Graduate School of Pure and Applied Sciences, University of Tsukuba, Tsukuba 305-8571, Japan

${ }^{c}$ Yukawa Institute for Theoretical Physics, Kyoto University, Kyoto 606-8502, Japan

${ }^{d}$ Department of Physics, Osaka University Toyonaka, Osaka 560-0043, Japan

e School of High Energy Accelerator Science, the Graduate University for Advanced Studies (Sokendai), Tsukuba 305-0801, Japan

We carry out numerical simulations of 2+1-flavor QCD with nearly chiral lattice fermions. Lattice spacing is taken at $a^{-1}=2.4$ and $3.6 \mathrm{GeV}$, while keeping the condition $m_{\pi} L \gtrsim 4$. Using the Möbius-type 5D implementation of the Ginsparg-Wilson fermion, the residual mass is always kept lower than $0.5 \mathrm{MeV}$. We report on our basic studies including a determination of lattice spacing through the Wilson flow as well as light hadron mass spectrum.

31 st International Symposium on Lattice Field Theory LATTICE 2013

July 29 - August 3, 2013

Mainz, Germany

\footnotetext{
* Speaker.

${ }^{\dagger}$ Email: noaki@ post.kek.jp
} 


\section{New simulation setup}

Motivated by increasing phenomenological importance of heavy flavor physics, we started a new set of lattice configuration generations at fine lattice spacings. For heavy quarks we are testing a new lattice formulation designed to suppress discretization effects [四], while for light sea/valence quarks we require good chiral symmetry to control possible systematic effects near the physical light quark masses. We use a variant of domain-wall fermions [ [ $[$, [ $]$, as described below, together with the tree-level Symanzik gauge action, for our 2+1-flavor gauge configuration generations.

Using the Wilson-Dirac operator $D_{W}(-M)$ with a negative mass parameter $-M$, our Dirac operator is defined by

$$
\begin{aligned}
& D_{\mathrm{DW}}^{5}(m)_{s s^{\prime}}= {\left[(4+M) D_{W}(-M)(b+c L(m))+1-L(m)\right]_{s s^{\prime}}, } \\
& L(m)_{s s^{\prime}}=\left\{\begin{array}{cl}
-m P_{+} \delta_{s^{\prime}, L_{s}}+P_{-} \delta_{s, s^{\prime}-1} & (s=1) \\
P_{+} \delta_{s, s^{\prime}+1}+P_{-} \delta_{s, s^{\prime}-1} & \left(1<s<L_{s}\right) \\
P_{+} \delta_{s, s^{\prime}+1}-m P_{-} \delta_{s^{\prime}, 1} & \left(s=L_{s}\right),
\end{array}\right.
\end{aligned}
$$

where $s$ and $s^{\prime}$ are indices of the 5 th direction of size $L_{s}$ and $m$ is the bare quark mass. Through a study of the residual quark mass [ [ 2 , [ $[$ ] , we choose parameters $b$ and $c$ as $b=2, c=1$ and $M=1.0$. With 3 steps of the stout smearing, the residual mass is suppressed to the level of $0.5 \mathrm{MeV}$ or better with $L_{s} \leq 12$. Other details of the numerical simulation are summarized in ref. [0]. We are developing a new code set called IroIro++ for this project [四].

The four-dimensional effective operator is constructed as

$$
D_{\mathrm{DW}}^{4}(m)=\left[\mathscr{P}^{-1} D_{\mathrm{DW}}^{5}(m=1)^{-1} D_{\mathrm{DW}}^{5}(m) \mathscr{P}\right]_{11},
$$

where $\mathscr{P}=\delta_{s s^{\prime}} P_{-}+\delta_{s+1\left(\bmod L_{s}\right), s^{\prime}} P_{+}$projects onto the four dimensional surfaces. For the 5D operator (ㅁ. $\mathbb{D}$ ) this leads to

$$
D_{\mathrm{DW}}^{4}(m)=\frac{1+m}{2}+\frac{1-m}{2} \gamma_{5} \tanh \left(L_{s} \tanh ^{-1}\left(\gamma_{5} \frac{b D_{W}}{2+c D_{W}}\right)\right),
$$

which satisfies the Ginsparg-Wilson relation in the limit of $L_{S} \rightarrow \infty$.

Table $\mathrm{W}$ lists gauge ensembles we are generating. We carry out main runs at a physical volume $\approx L^{3}=(2.6 \mathrm{fm})^{3}$. Two values of the gauge coupling $\beta=4.17$ and 4.35 correspond to $a^{-1} \approx 2.4$ and $3.6 \mathrm{fm}$, respectively, and we are planning to generate even finer lattices in the future. The extrapolation to the physical light quark mass is made from the data in the range of $240 \mathrm{MeV}<m_{\pi}<500$ $\mathrm{MeV}$. The physical strange quark mass is sandwitched by two values of $m_{s}$. We designed our main runs to satisfy $m_{\pi} L \gtrsim 4$, hence the artifact of finite volume is under good control. Simulation with a small $m_{\pi} L$ is also done at the lightest mass $m_{\mathrm{ud}}=0.0035$ with $L=32$ for a direct study of the finite volume effect. In the last two columns of the table, we summarize the number of generated HMC trajectories after thermalization with unit MD length of $\tau=1$ and 2. A check of the thermalization and the motivation for adding the $\tau=2$ runs will be explained in Sec. . . The analyses we present in this article are all from the $\tau=1$ runs. 


\begin{tabular}{|c|c|c|c|c|c|c|c|}
\hline$\beta$ & $L_{5}$ & size & $m_{\mathrm{s}}$ & $m_{\mathrm{ud}}$ & $m_{\pi}[\mathrm{MeV}]$ & $\#$ trajs $(\tau=1)$ & \#trajs $(\tau=2)$ \\
\hline \multirow[t]{8}{*}{4.17} & 12 & $32^{3} \times 64$ & 0.030 & 0.0070 & 310 & 3,000 & - \\
\hline & & & & 0.0120 & 400 & 3,000 & - \\
\hline & & & & 0.0190 & 500 & 3,000 & - \\
\hline & & $32^{3} \times 64$ & 0.040 & 0.0035 & 240 & 3,000 & - \\
\hline & & & & 0.0070 & 310 & 3,000 & - \\
\hline & & & & 0.0120 & 400 & 3,000 & - \\
\hline & & & & 0.0190 & 500 & 3,000 & - \\
\hline & & $48^{3} \times 96$ & 0.040 & 0.0035 & 240 & 1,500 & - \\
\hline \multirow[t]{6}{*}{4.35} & 8 & $48^{3} \times 96$ & 0.018 & 0.0042 & 290 & 500 & 280 \\
\hline & & & & 0.0080 & 410 & 1,800 & 260 \\
\hline & & & & 0.0120 & 500 & 2,000 & - \\
\hline & & $48^{3} \times 96$ & 0.025 & 0.0042 & 290 & 700 & 235 \\
\hline & & & & 0.0080 & 410 & 680 & 330 \\
\hline & & & & 0.0120 & 500 & 1,550 & 430 \\
\hline
\end{tabular}

Table 1: Profiles of the generated gauge ensembles. The last two columns show the number of the thermalized trajectories for $\tau=1$ and $\tau=2$ which have been accumulated.

\section{Scale setting by the Wilson flow}

We determine the lattice scale $a^{-1}$ of the generated configurations through the Yang-Mills gradient flow []], which is an evolution of gauge configuration $V_{x \mu}(t)$ in a fictitious time $t$ following

$$
\left.\frac{d V_{x \mu}}{d t}\right|_{t}=-g_{0}^{2} \partial_{x \mu} S_{g}[V], \quad V_{x \mu}(0)=U_{x \mu} .
$$

Here $g_{0}$ is the bare gauge coupling and $S_{g}$ is the Wilson gauge action. In steps of 10 trajectories, we compute the energy density $E=\frac{1}{4} F_{\mu \nu}^{a} F_{\mu \nu}^{a}$ evaluated on $\left\{V_{x \mu}\right\}$ at a reference point $t_{0}$ or $w_{0}$ satisfying the conditions [[1, 目]

$$
\left.t^{2}\langle E\rangle\right|_{t=t_{0}}=0.3,\left.\quad t \frac{d}{d t}\left(t^{2}\langle E\rangle\right)\right|_{t=w_{0}^{2}}=0.3
$$

To reduce discretization effects in $E$, we use its improved definition with the clover-leaf terms. In Figure 四, data of $t^{2}\langle E\rangle$ are shown for $\beta=4.17, m_{s}=0.030$ (left) and 4.35, $m_{s}=0.018$ (right). Although $w_{0}$ at larger $t$ region is supposed to suffer less from cutoff effects than $t_{0}$ does, we use $t_{0}$ to determine the lattice spacing in our preliminary study as it is more stable as a function of the quark mass. As a preliminary result, we obtain

$$
\beta=4.17: a^{-1}=2.48(1) \mathrm{GeV}, \quad \beta=4.35: a^{-1}=3.68(1) \mathrm{GeV},
$$

where we only quote statistical errors. Study of other systematics such as finite size effect are to be done with improved statistics in the future. 

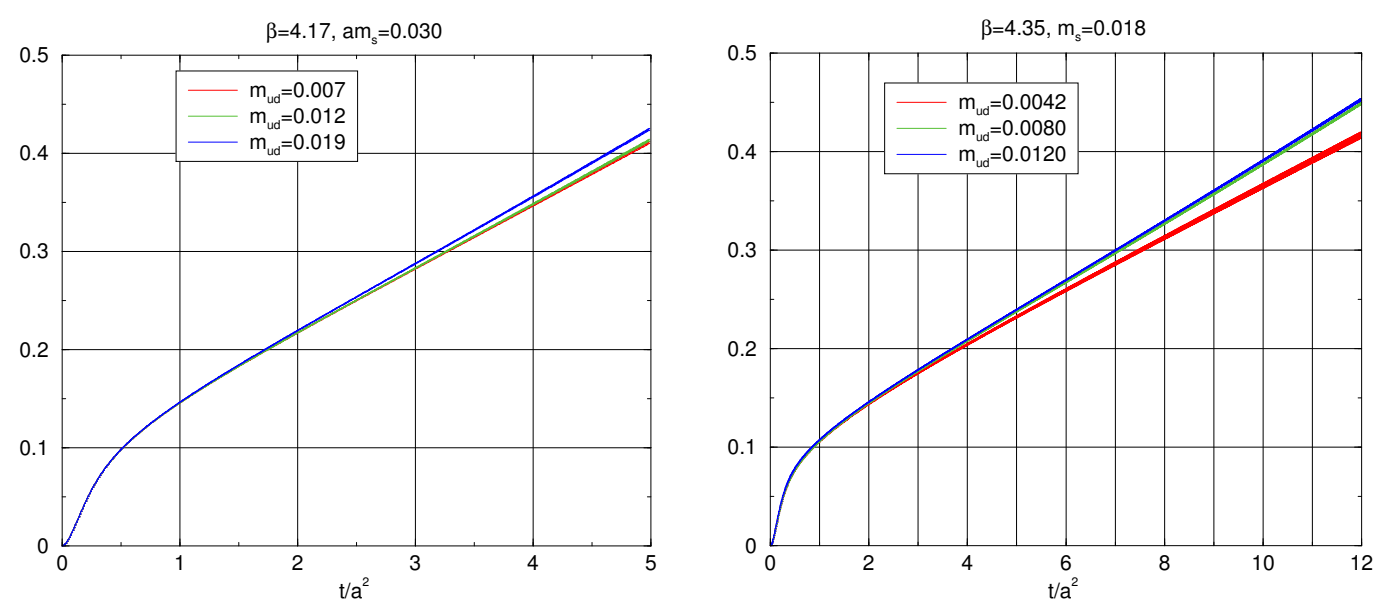

Figure 1: $t^{2}\langle E\rangle$ as a function of the flow time $t$. Side by side, data from $\beta=4.17$ and $m_{\mathrm{s}}=0.030$ (left) $\beta=4.35$ and $m_{\mathrm{s}}=0.018$ (right) are shown. In each panel, different colors correspond to different gauge ensembles with different $m_{\mathrm{ud}}$.

\section{Light hadron spectrum with the all mode averaging}

On the generated configurations, we compute quark propagator

$$
\left\langle q_{x} \bar{q}_{y}\right\rangle=\frac{1}{1-m}\left(D_{\mathrm{DW}}^{4}(m)_{x y}^{-1}-\delta_{x y}\right)
$$

where an inverse of the 4D-operator is obtained through eq. ([L.3)). We use a source vector $e^{-\alpha r}$ in the Coulomb gauge as a function of the distance $r$ from the center of the source with $\alpha=0.4$ and 0.2 for $\beta=4.17$ and 4.35 , respectively. Since the statistical error in the hadron two-point correlation functions is large with our limited samples, we use the all-mode-averaging (AMA) technique [ $[\mathbb{}]$, where, besides the data with the desired precision $C(t)$, we generate $C^{\text {bulk }}(t)$ with a relaxed precision at as many source locations as possible. $C(t)$ is improved by averaging over $N_{s}$ and $N_{s}^{\text {bulk }}$ time-slices for the regular precision part and for the bulk part as

$$
C(t) \rightarrow \frac{1}{N_{s}} \sum_{t_{s}=1}^{N_{s}}\left(C\left(t ; t_{s}\right)-C^{\text {bulk }}\left(t ; t_{s}\right)\right)+\frac{1}{N_{s}^{\text {bulk }}} \sum_{t_{s}=1}^{N_{s}^{\text {bulk }}} C^{\text {bulk }}\left(t ; t_{s}\right),
$$

where, in the right hand side, we explicitly indicate the source location $t_{s}$ as well as the time distance $t$ from the source location. The averaging in the second term requires a lower cost than the conventional one because of the relaxed convergence condition.

On every 10 trajectories of the $\beta=4.17$ configurations of size $32^{3} \times 64$, we take $N_{s}=2$ and $N_{s}^{\text {bulk }}=32$ equally separated time-slices. The number of CG-iterations for each solve of $C^{\text {bulk }}(t)$ is $\approx 10 \%$ of that of $C(t)$ 's and the total cost for $N_{s}^{\text {bulk }}=32$ is compatible with that of the $N_{s}=2$ inversions with the regular precision. In Figure $\square$, the effective masses of pseudo-scalar (upper left), vector (upper right) mesons and nucleon (lower) at $m_{\mathrm{s}}=0.030$ are shown. The different colors correspond to different $m_{\mathrm{ud}}$ 's. In each panel, we compare the case with AMA (eq. (B.2), filled symbols) and without (i.e. $\frac{1}{N_{s}} \sum_{t_{s}} C\left(t ; t_{s}\right)$, open symbols). We observe that the plateau is 


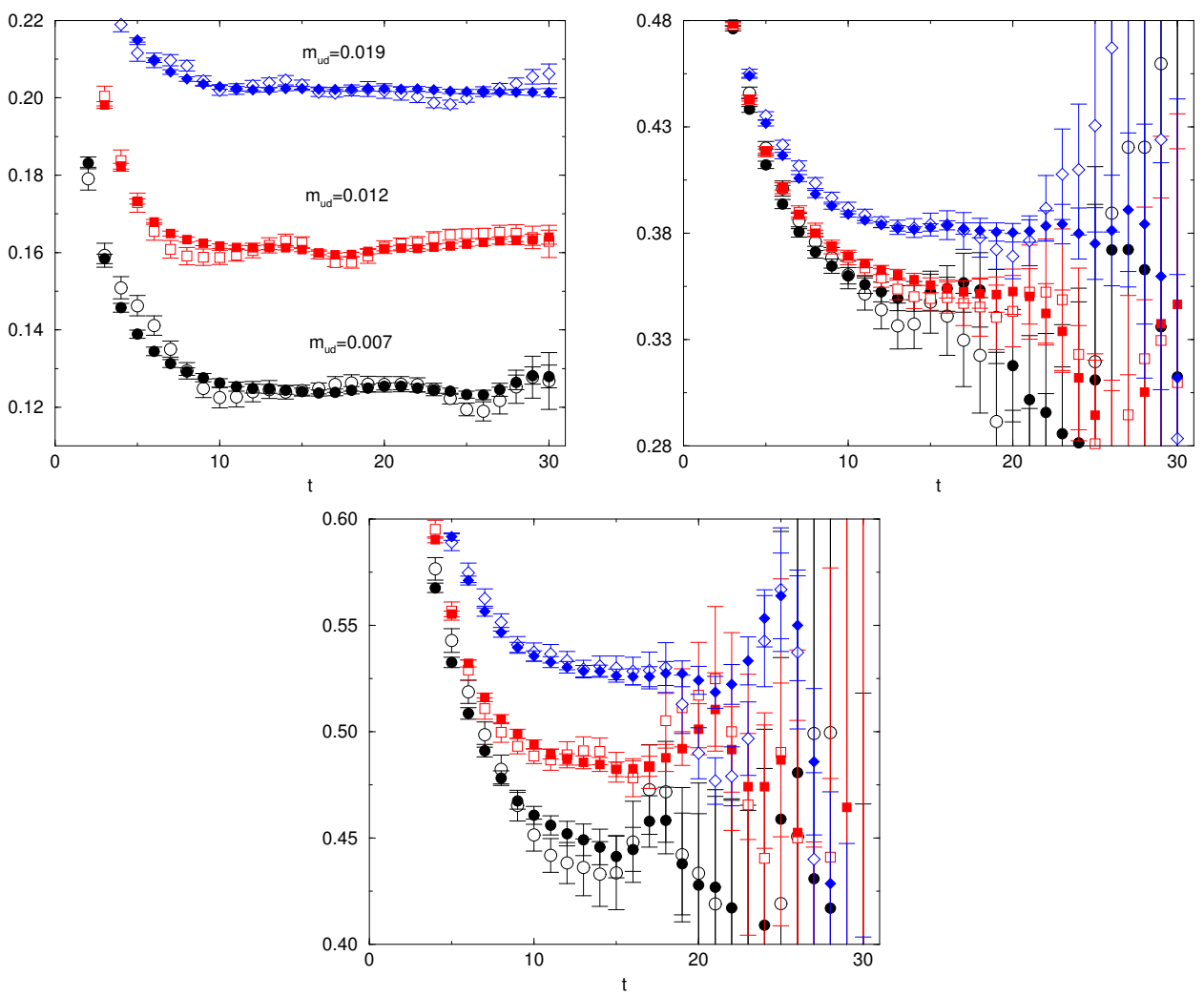

Figure 2: Effective mass plots of pseudo-scalar (upper left), vector (upper right) mesons and nucleon (lower) at $\beta=4.17$ and $m_{\mathrm{s}}=0.030$. Data with the all-mode-averaging applied (filled) are compared with the conventional ones (open) in each panel.

visible with AMA in the $t$ region of $t \gtrsim 12$. With the total costs which is about three times more than the case with the conventional method $\left(N_{s}=2\right)$, the gain amounts to $30-50 \%$ decrease of the statistical error.

An important question to address is how large $N_{s}^{\text {bulk }}$ is really needed to obtain the signal at the level of that with $N_{s}^{\text {bulk }}=32$. We examined the size of error for the vector-meson effective mass at several time-slices by repeating the same analyses with $N_{s}^{\text {bulk }}=4,8$ and 16 , and found a saturation to start at $N_{s}^{\text {bulk }}=8$. Based on this observation, the measurement on the $\beta=4.35$ configurations are being done with $N_{s}^{\text {bulk }}=8$, i.e. at time-slices in steps of 12 .

\section{Thermalization and auto-correlation}

Since we are generating gauge configurations at a fine lattices especially for the fine lattice at $\beta=4.35$, the issue of critical slowing down, i.e. long thermalization and auto-correlation may arise. Before going to more advanced physics analysis, a detailed study is necessary.

A simple way to check the thermalization is to monitor the plaquette on generated configurations, which is shown in the light panels of Figure [3. Upper and lower panels are those for two smallest ud-quark masses respectively. From them, the configurations seem to be thermalized in both cases. On the other hand, the histories of the CG-iteration counts in the right panels seem to 

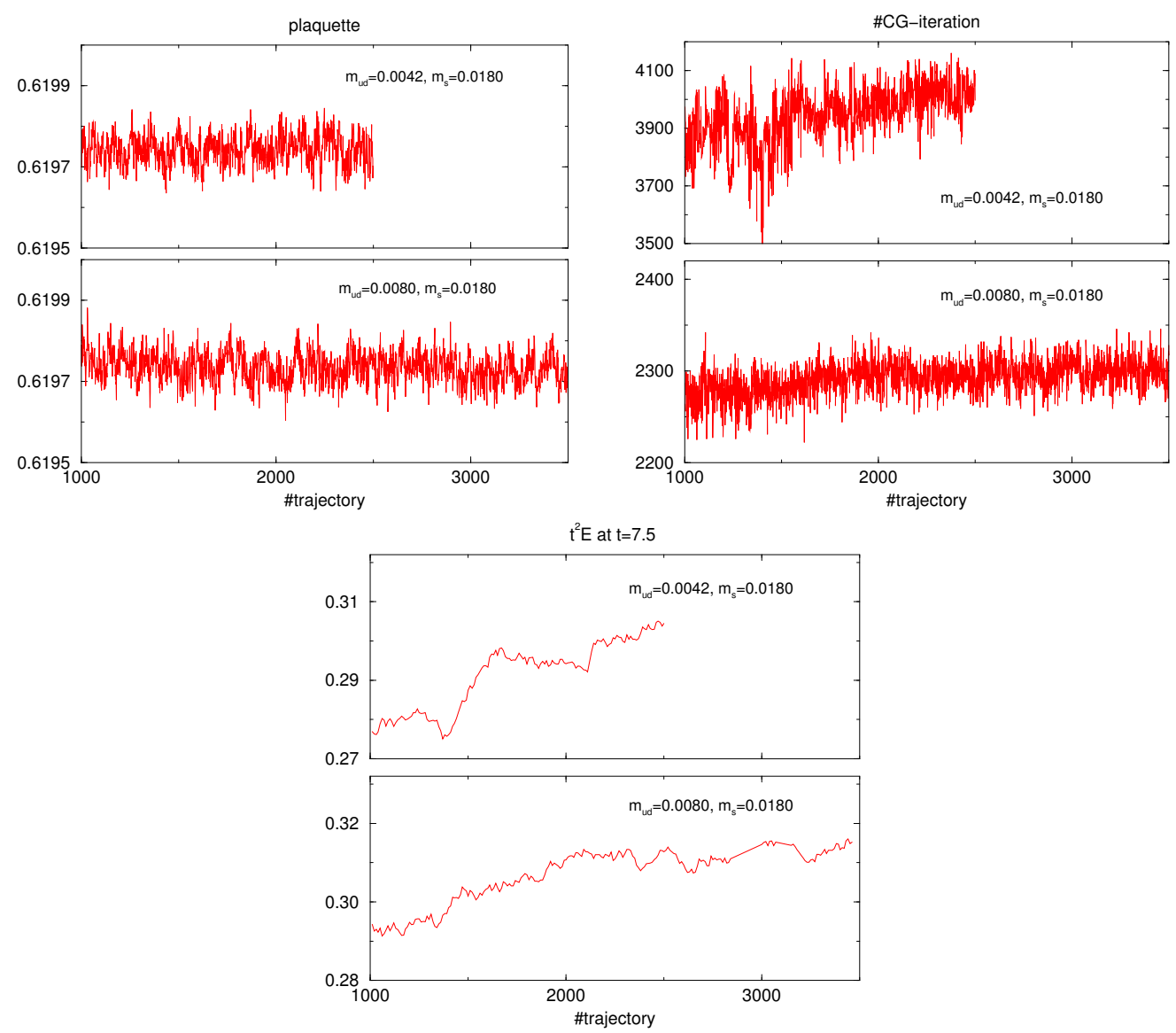

Figure 3: HMC histories of plaquette (upper left panels), CG-iteration count (upper right) and $t^{2} E$ at $t=7.5$ for a $\beta=4.35$ run (bottom). Data of the lightest two $m_{\mathrm{ud}}$ 's with $m_{\mathrm{s}}=0.018$ are compared between upper and lower panels for each quantity.

be increasing in the same trajectory region especially before 2,000 . Such a long thermalization process is more prominent when the dimensionless energy density $t^{2}\langle E\rangle$ on the Yang-Mills gradient flow (at $t=7.5$ ) is monitored (bottom panels). We also looked at the values of hadron correlators at specific time-slices. By using the data measured on the configurations in question, we examined pion, $\rho$, nucleon and $\Omega$. Except for pion, we found that the correlators are monotonically drifting in the same region of trajectories which become more significant at the time-slices far apart from the source. In other words, these quantities are sensitive to the thermalization and auto-correlation. We also checked thermalizations and autocorrelations for the $\beta=4.17$ data and found no such non-trivial behavior.

To partly cure the long auto-correlation problem, we doubled the MD trajectory length $(\tau=2)$ followed by the suggestion given in ref. [8]. The numbers of accumulated new trajectories at the

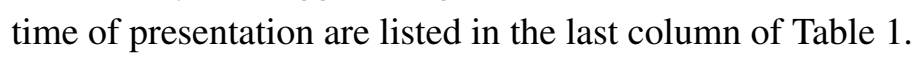




\section{Outlook}

Plotting the results of $m_{\pi}, m_{K}$ and $m_{\eta_{s}}$ obtained with the current data, we find that the PCAC relation is reasonably satisfied. On the other hand, we need more statistics before studying the chiral properties of these masses and other basic observables such as $f_{\pi}, f_{K}$ and nucleon masses.

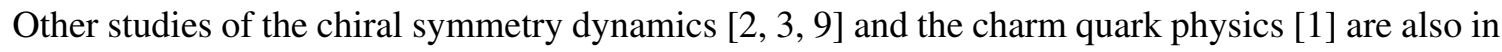
progress.

Numerical simulations are performed on the IBM System Blue Gene Solution at High Energy Accelerator Research Organization (KEK) under a support of its Large Scale Simulation Program (No. 12/13-04). This work is supported in part by the Grant-in-Aid of the Japanese Ministry of Education (Nos. 21674002, 25287046, 25800147) and the SPIRE (Strategic Program for Innovative Research) Field5 project.

\section{References}

[1] Y-G. Cho, S. Hashimoto and J. Noaki, $O\left(a^{2}\right)$-improved actions for heavy quarks, in these proceedings.

[2] T. Kaneko, S. Aoki, G. Cossu, H. Fukaya, S. Hashimoto and J. Noaki, Large-scale simulations with chiral symmetry, in these proceedings.

[3] S. Hashimoto, S. Aoki, G. Cossu, H. Fukaya,T. Kaneko and J. Noaki, Residual mass in five-dimensional fermion formulations, in these proceedings.

[4] G. Cossu, J. Noaki, S. Hashimoto, T. Kaneko, H. Fukaya, P.A. Boyle and J. Doi, JLQCD IroIro++ lattice code on $B G / Q$, in these proceedings.

[5] M. Lüscher, Properties and uses of the Wilson flow in lattice QCD, JHEP 08 (2010) 071.

[6] S. Borsanyi et. al. (BMW Collaboration), High-precision scale setting in lattice QCD, JHEP 09 (2012) 010 .

[7] T. Blum, T. Izubuchi and E. Shintani A new class of variance reduction techniques using lattice symmetries, arXiv:1208.4349 [hep-lat].

[8] B. Blossier et. al. (ALPHA Collaboration), Parameters of Heavy Quark Effective Theory from $N_{f}=2$ lattice QCD , JHEP 09 (2012) 132.

[9] H. Fukaya, S. Aoki, G. Cossu, S. Hashimoto, T. Kaneko and J. Noaki, Overlap/Domain-wall reweighting, in these proceedings. 